\title{
La fortuna del Filocolo in Francia nel secolo XVI
}

\section{Silvia D'Amico}

\section{OpenEdition Journals}

Edizione digitale

URL: http://journals.openedition.org/cei/899

DOI: 10.4000/cei.899

ISSN: 2260-779X

\section{Editore}

UGA Éditions/Université Grenoble Alpes

\section{Edizione cartacea}

Data di pubblicazione: 15 luglio 2008

Paginazione: 195-207

ISBN: 978-2-84310-122-9

ISSN: $1770-9571$

Notizia bibliografica digitale

Silvia D'Amico, « La fortuna del Filocolo in Francia nel secolo XVI », Cahiers d'études italiennes [Online], 8 | 2008, Messo online il 15 janvier 2010, consultato il 01 mai 2019. URL : http:// journals.openedition.org/cei/899; DOI : 10.4000/cei.899 


\title{
LA FORTUNA DEL FILOCOLO \\ IN FRANCIA NEL SECOLO XVI
}

\author{
Silvia D'Amico \\ Université de Savoie-Chambéry
}

Scrisse egli il Filocopo e puosevi quanta industria e arte seppe per averlo dedicato alla reina Gioanna da lui amata: consideri (prego) qualunque è di pazienza ben armato se al mondo legger si possi libro di maggior fastidio. Credo io ch'egli nascesse ad un parto col tedio. Tutte le volte ch'io lo piglio nelle mani per leggerlo subito mi s'instechiscono le dita, di maniera che forza è che dalle mani mi cada. Diceva già un mio sviscerato amico (non gli farò il nome per non provocargli contra alcuno di questi spensierati bocacceschi) che con maggior sofferenza sostenuto avrebbe d'esser trafitto da mosche, da taffani e da zenzare che di continovar un sol giorno in sì stomacosa lezione.

Questo « rabbuffamento » al Boccaccio, come ebbe a definirlo, divertito, il Doni in una lettera del $1544^{1}$, è contenuto nei Paradossi di Ortensio Lando, opera originale per gli argomenti provocatori e controcorrente, pubblicata a Lione nel $1543^{2}$.

Nel loro esibito anticonformismo, i Paradossi si rivelano in realtà uno specchio fedele e prezioso della doxa letteraria del loro tempo e confermano quello che è un dato di partenza indiscutibile per la ricognizione della fortuna del Filocolo in Francia nel Rinascimento: il primo romanzo di Boccaccio e della letteratura italiana a partire dagli anni '30 godette oltralpe di grande considerazione, era giudicato come un "classico", un

1. Antonio Francesco Doni, Lettere, Venezia, Girolamo Scotto, 1544, cc. 116r-117r. La lettera, indirizzata ad Alessandro Giovio, è citata in Ortensio LANDO, Paradossi cioè sentenze fuori del comun parere, a cura di A. Corsaro, Roma, Edizioni di storia e letteratura, 2000, p. 11.

2. Ortensio Lando, Paradossi, cit., pp. 243-244. Il libro del Lando offre un punto di vista privilegiato per gli studi sugli scambi tra Italia e Francia nel Cinquecento : il suo percorso editoriale fu infatti anomalo, per così dire à l'envers, con una prima edizione in italiano in Francia, a Lione, dove nel 1543 una traduzione parziale circolava ancor prima dell'edizione a stampa, a testimoniare dell'attenzione dei lettori - e non i più oscuri, vista l'identità del traduttore, cioè Maurice Scève. Cfr. Verdun-Louis SaUlnier, Maurice Scève, Genève, Slatkine, 1981 (ristampa dell'edizione di Parigi, 1948-1949), pp. 117-119. 
vademecum indispensabile per l'uomo e per la donna di corte che aspirassero a una qualche reputazione letteraria e, quindi, sociale.

Già da una semplice costatazione dei primi dati oggettivi (il numero e le date delle traduzioni, i formati delle edizioni, le illustrazioni) lo studio della ricezione del Filocolo in Francia si annuncia promettente e non puramente aneddotico. Il caso del Filocolo sembra infatti essere uno di quelli in cui la fortuna in terra straniera può mettere in luce delle potenzialità del testo che - per varie ragioni - restano inespresse nel paese d'origine e, nel contempo, seguire la fortuna del testo italiano in Francia può offrire un punto di vista realmente inedito che permette di cogliere più nitidamente scenari che non si possono percepire limitandosi alla prospettiva francese.

Il rifacimento in prosa del contrastato amore di Florio e Biancofiore si diffonde in Francia in due fasi che seguono strade indipendenti, sia pure con qualche significativo punto di tangenza: la prima ha inizio nel 1531 con l'edizione della traduzione anonima del celebre episodio delle Questioni d'amore ${ }^{3}$. Le Treize élégantes demandes d'amour hanno, nel volgere di un decennio, l'onore di altre due edizioni, mentre nel $1542-\mathrm{e}$ siamo alla seconda fase della fortuna - viene stampata la traduzione completa del Filocolo ad opera di Adrien Sévin, con lo stesso formato e illustrazioni dei primi libri degli Amadigi. La traduzione di Sévin ebbe un certo successo, come provano le edizioni successive fino al $1575^{4}$.

Questi primi dati bastano ad assicurare al Filocolo un posto d'onore in due dei capitoli più significativi della storia letteraria del XVI secolo francese: la produzione relativa al tema amoroso proveniente dall'Italia e la nascita e lo sviluppo del romanzo, legato al fenomeno editoriale degli Amadigi.

\section{Le Treize questions}

Contenuto nel quarto libro del Filocolo, l'episodio delle Tredici questioni viene recepito fin dalle prime fasi della circolazione del romanzo in Italia

3. Pio RajNA, L'episodio delle "questioni d'amore" nel Filocolo del Boccaccio, in "Romania", XXXI, 1902, pp. 28-81 (ora in IDEM, Scritti di filologia e linguistica italiana e romanza, a cura di G. Lucchini, Roma, Salerno, 1998, t. II, pp. 671-727; le mie citazioni sono tratte da questo volume); Victoria KIRKHAM, Reckoning with Boccaccio's "Questioni d'amore», in "Modern Language Notes", 89, 1974, pp. 47-59; Paolo CHERCHI, Andrea Cappellano, i trovatori e altri temi romanzi, Roma, Bulzoni, 1979, pp. 210-217; Luigi Magaloli, Timbro della prosa e motivi dell'arte nel Filocolo, in «Studi mediolatini e volgari», VI-VII, 1959, pp. 105-111.

4. L'elenco delle edizioni delle traduzioni francesi si trova in Alberto BACCHI Della LeGA, Bibliografia boccaccesca. Serie delle edizioni delle opere di G. Boccaccio, Bologna, Forni, 1875, pp. 107-108. 
come segmento isolabile del testo, come si evince dalla tradizione manoscritta. L'idea di separare l'episodio dal resto del romanzo nacque in Italia nel Quattrocento5. Questa forma ridotta del testo doveva essere diffusa, come prova il fatto che ancora oggi è possibile reperire manoscritti relativi al quarto libro di cui si era persa traccia o che non si conoscevano ancora: una nota pubblicata nel numero XXXI degli Studi sul Boccaccio, per esempio, segnala un manoscritto del XV secolo messo all'asta a Venezia nel maggio $2003^{6}$. Il dato importante su cui riflettere dal punto di vista della ricezione europea è il fatto che solo all'estero, in Francia, in Spagna e in Inghilterra, le Tredici questioni diventano oggetto di un'edizione a stampa isolata dal resto del romanzo ${ }^{7}$.

Cercare di capire perché in Francia si avverte l'esigenza di stampare l'episodio delle "Questioni d'amore», a quale gusto del tempo questo testo, antico di duecento anni, poteva dare ancora una risposta attuale, a quale pubblico si rivolgeva, costituisce lo scopo delle riflessioni che seguono.

L'attenzione per le dispute d'amore, come ha osservato Gustave Reynier nel noto libro Le Roman Sentimental avant l'Astrée, è viva in Francia a partire dal Medioevo, come si evince, per esempio, dalle reiterate edizioni degli Arrest d'Amour di Martial d'Auvergne ${ }^{8}$. Questo gusto per il dibattito amoroso si precisa all'inizio del Cinquecento: le questioni d'amore assurgono infatti a gioco di corte usuale e inevitabile, tanto da essere evocate, con sprezzatura ovviamente, come abitudine del tutto naturale, nel luogo paradigmatico per eccellenza del comportamento cortese del Rinascimento, cioè all'inizio del primo libro del Cortigiano:

5. Cfr. P. RajNA, «L'episodio delle questioni d'amore», cit., p. 671. Per la tradizione manoscritta del Filocolo si veda Vittore BRANCA, Tradizione delle opere di Giovanni Boccaccio, Roma, Edizioni di Storia e di Letteratura, 1958, pp. 37-40.

6. "Studi sul Boccaccio», XXXI (2003), p. 27. Il regesto riporta: "Questo codice contiene le tredici quistioni d'amore disputate e risolute nel $5^{\circ}$ Libro del Filocolo di Messer Giovanni Boccaccio. Per maggior intelligenza chi le compilò in questo volume fece in principio un compendio degli accidenti descritti in quest'opera dal Boccaccio e lo stesso ancora in un breve capitolo aggiunto in fine. Il testo del Boccaccio comincia e corrisponde alla pag. 404 dell'edizione del Filocolo del 1594 de' Giunti, e continua fino presso alla fine di tutto il $5^{\circ}$ Libro. E'scritto assai correttamente, e sembra carattere del secolo $1400 \mathrm{ma}$ indizio veruno ne dello scrittore ne del professore $m$. apparisce».

7. P. RAJNA, «L'episodio delle questioni d'amore», cit., pp. 671-679; Henri HaUvetTe, Les plus anciennes traductions françaises de Boccace, Bordeaux, Feret et fils, 1909, pp. 2-18.

8. Gustave ReYnier, Le Roman Sentimental avant l'Astrée, Paris, Armand Colin, 1908, p. 48. Si veda anche V.-L. SAULnier, Maurice Scève, cit., p. 50. 
Ma lasciando questo, dico che consuetudine di tutti i gentiluomini della casa era ridursi subito dopo cena alla signora Duchessa, dove, tra le altre piacevoli feste e musiche e danze che continuamente si usavano, talora si proponevano belle quistioni, talora si facevano alcuni giochi ingegnosi ad arbitrio ora di uno ora di un altro, nei quali sotto vari velami spesso scoprivano i circostanti allegoricamente i pensieri suoi a chi più loro piaceva. $(I, 5)$

Sappiamo quanto il Cortigiano influenzi i comportamenti della corte di Francesco I negli anni '30. Allenarsi nelle dispute attraverso la lettura diventa quasi un obbligo mondano e non desta quindi alcuno stupore la coincidenza tra il diffondersi del Cortigiano come libro simbolo della nuova società e della cultura ideale cui si vuole dare corpo e la commissione della traduzione francese e delle ripetute edizioni in formato elegante del quarto libro del Filocolo, destinato anche al sempre più esigente pubblico femminile della corte. Per la legge delle aree periferiche, il Cortigiano finisce per essere un modello di comportamento più coercitivo all'estero che in Italia: i giochi di corte a sfondo letterario svolgono un ruolo fondamentale nell'ispirazione dei testi in poesia e in prosa, finendo per coinvolgere perfino il re e le persone a lui più vicine. La pubblicazione della traduzione dell'episodio delle Questioni d'amore va inserita nella storia della fortuna di queste composizioni a soggetto amoroso provenienti dall'Italia?.

Copie manoscritte del Filocolo erano presenti in Francia fin dal Quattrocento: due manoscritti sono conservati alla Bibliothèque Nationale ${ }^{10}$; un manoscritto del XV secolo, ora perduto, figurava tra i libri di casa Savoia nella Biblioteca di Chambéry ${ }^{11}$ : è verosimile ipotizzare che facesse parte dell'eredità di Luisa di Savoia e che fosse quindi facilmente accessibile per i suoi figli. È l'esigenza di disporre di una traduzione che ispira in Francia il progetto di un'edizione isolata delle Questioni. Nell'ambiente di corte, dove nei primi decenni del Cinquecento il Filocolo era conosciuto quindi sia attraverso il manoscritto, sia attraverso le edizioni a stampa in italiano (la Bibliothèque Nationale ne conserva diversi esemplari $\left.{ }^{12}\right)$, si riconosce il capitolo delle Questioni d'amore come il più

9. Cfr. Lionello SozzI, «Boccaccio in Francia nel Cinquecento", in Il Boccaccio nella letteratura francese, a cura di C. Pellegrini, Firenze, Olschki, 1971, p. 258.

10. Si veda l'elenco dei codici del Filocolo in V. BrancA, Tradizione delle opere..., cit., p. 38.

11. Cfr. V. BrancA, Tradizione delle opere..., cit., p. 40; Gianni Mombello, «I manoscritti di Dante, Petrarca e Boccaccio in Francia", in Il Boccaccio nella cultura francese, cit., p. 158.

12. 5 incunaboli e 8 cinquecentine. 
affine al gusto e alle attività di corte (la cornice del racconto, le novelle e, soprattutto, la riflessione sulla casistica amorosa) e si avverte dunque il desiderio di leggerlo in francese. In Italia negli anni '30 è meno forte la moda delle Questioni, ma soprattutto il facile accesso all'edizione completa fin dal 1472 rende meno urgente la pubblicazione separata del segmento.

Delle tre edizioni delle Treize elegantes demandes d'amour, quella del 1541 conservata alla Bibliothèque de l'Arsenal di Parigi ${ }^{13}$, presenta alcuni aspetti molto interessanti sui quali vale la pena di soffermarsi. L'editore Denis Janot ripubblica la traduzione anonima del quarto libro del Filocolo, in un formato in- $16^{\circ}$, con una prefazione e, dato più appariscente, con quindici illustrazioni. Henri Hauvette, nel suo ampio e dettagliato saggio sulle prime traduzioni francesi di Boccaccio ${ }^{14}$, segnala la presenza di queste 15 "vignettes", sulle quali però non dà alcuna informazione. Ora, alcune di queste "vignettes», quattro per la precisione $^{15}$, sono le stesse già utilizzate da Galliot du Pré per Les Fleurs de Poesie Françoyse del 1534 e dallo stesso Denis Janot per Les Angoisses douloureuses qui procèdent d'amour di Hélisenne de Crenne nel $1538^{16}$. Il libro in quanto 'oggetto', quindi, viene concepito come assolutamente affine a una pubblicazione che è un gioco di corte su soggetti amorosi: (Les Fleurs de Poesie Françoyse, una raccolta di poesia collettiva cui, dietro lo schermo cortese di pseudonimi trasparenti per i contemporanei, partecipano i maggiori poeti del momento - Clément Marot in testa, e personaggi di primo piano, è il minimo che si possa dire, visto che si tratta di Francesco I e Margherita di Navarra, come ha dimostrato Gérard Defaux nella sua edizione del $2001^{17}$. Il libro viene anche pensato come strutturalmente e funzionalmente simile a uno dei più importanti romanzi francesi di argo-

13. Treize élégantes demandes d'amours, premièrement composées par... Jehan Boccace, et depuis translatées en françoys..., Paris, D. Janot, 1541, Bibl. de l'Arsenal, $8^{\circ}$ B.L. 32714.

14. H. HAUVETTE, Les plus anciennes traductions françaises, cit., p. 4.

15. La terza, foglio XVIv ${ }^{\circ}$ (che ritorna anche al foglio XLVIIv ${ }^{\circ}$ ); la settima, foglio XXXVIIr ${ }^{\circ}$; la nona, foglio XLIIIIv ${ }^{\circ}$ e la decima, foglio XLVIr ${ }^{\circ}$. Non farò una descrizione esaustiva del ricorrere di queste illustrazioni in tutti i libri dell'epoca. Per spiegare le mie osservazioni basterà un esempio: la terza figura delle Treize elegantes demandes d'amour (che rappresenta un uomo e una donna nell'atto di conversare) è la stessa utilizzata ne Les angoysses douloureuses qui procedent d'amour: contenantz troys parties, composees par Dame Helisenne..., Paris, Denis Janot, s.d. [1538]) (v. p. 29 e p. 80 del formato Pdf; Bnf: Res. P-Z-2013; NUMM-70526). Sempre questa stessa illustrazione si ritrova in Hecatomphile. De vulgaire Italien tourné en langaige François. Les fleurs de Poesie Françoise, Paris, Galliot du Pré, 1534 (p. 10 e 72 dell'edizione Les Fleurs de Poesie Françoyse. Hécatomphile, texte établi, présenté et annoté par G. Defaux, STFM, 2002).

16. Les angoysses douloureuses qui procedent d'amour, cit.

17. Les Fleurs de Poesie Françoyse, cit. 
mento amoroso, Les angoisses douloureuses, ispirato a un'altra opera "minore" di Boccaccio, che stava avendo in Francia un enorme successo proprio in questo decennio, com'è noto, e cioè la Fiammetta ${ }^{18}$. Questa prossimità, vera e propria identità, dell'immaginario nella concezione materiale del libro va sottolineata: l'editore propone al pubblico prodotti coerenti, si può dunque asserire con semplicità che la stessa corte che si divertiva a scambiarsi domande e risposte attraverso composizioni poetiche ispirate per lo più a Ovidio e Petrarca costituiva un pubblico avido di assaporare in francese il piacere della prosa di Boccaccio relativa alla casistica amorosa. Il formato in $-16^{\circ}$ e le illustrazioni fanno pensare, come abbiamo detto, al crescente pubblico femminile, che ritrovava nella traduzione del frammento del Boccaccio gli stessi temi su cui si fondavano sia le raccolte poetiche alla moda, sia il romanzo di Hélisenne de Crenne: l'innamoramento, la sofferenza amorosa, la gelosia.

Un altro aspetto di questa edizione che non può non attirare l'attenzione è la prefazione dell'anonimo traduttore ("Prologue du traducteur anonyme des Treize elegantes demandes d'amour de Jehan Boccace»). Vengono infatti evocate quattro figure mitiche, Dafne, Orfeo, Narciso e Circe come esemplari dei quattro tipi di amore: l'amore puro e sublime, Dafne, che consente al traduttore anche un prevedibile riferimento a Petrarca ${ }^{19}$; l'amore che resiste oltre la morte, Orfeo; l'amore che si nega, Narciso e, infine, l'amore che si limita alla sfera dei sensi, abbassando l'uomo allo stato selvaggio, di cui è simbolo Circe. Dopo essersi paragonato all'eloquente Ulisse, il traduttore afferma di voler dedicare la sua traduzione all'unica donna che meriti lodi celesti, sperando che possa il suo omaggio esprimere quello che mai fu detto di alcuna, con riferimento inequivocabile alla chiusa della Vita Nuova, testo la cui conoscenza in quegli anni in Francia non era affatto scontata. Questo dato, come suggerisce Hauvette $^{20}$, attira la curiosità dello studioso sull'identità del traduttore, la

18. Sulla fortuna della Fiammetta in Francia nel Cinquecento si veda H. HauvetTe, Les plus anciennes traductions, cit. pp. 33-43, V.-L. SAULNIER, Maurice Scève, cit., pp. 49-71. Si veda anche il contributo di Serge Stolf in questo volume.

19. "Ceste cy fut choysie pour unique maistresse par l'eloquent Florentin Petrarcque, et fut par elle conduict iusques a contempler les sept excellentz triumphes qu'il nous a laissez par escript. Ceste ci a este esleue par les nobles esperitz qui sans eulx arrester aux blandices venerees ont prefere l'honneur de leurs maistresses a toutes delices, et repute les refus du libidineux plaisir a constante vertu verdoyant comme le perpetuel laurier desprisans la fureur de tous medisans». Il riferimento a Petrarca era sua volta un elemento di attrattiva per il pubblico. Sul fenomeno del petrarchismo in Francia vedi Les poètes français de la Renaissance et Pétrarque, études réunies par J. Balsamo, Genève, Droz, 2004.

20. Les plus anciennes traductions, cit., p. 5. 
quale, però resta ancora da accertare. La prefazione contiene anche un riferimento a Ovidio ${ }^{21}$, altro autore la cui enorme fortuna nel contesto sociale della corte si interseca e si sovrappone alla ricezione del Filocolo ${ }^{22}$.

La ricettività della cultura francese rispetto al tema delle questioni d'amore è attestata anche da un altro dato: nello stesso 1541 viene pubblicata, sempre da Denis Janot, la traduzione del romanzo spagnolo Cuestiòn de Amor ${ }^{23}$, la cui prima edizione, in Spagna, risaliva al 1513. Questo testo riprende sotto forma di novella il quesito già affrontato dal Boccaccio in una delle questioni, se soffre di più colui che perde l'oggetto del proprio amore dopo averlo posseduto oppure chi, essendo respinto, non riesce a goderne affatto. Il legame fra questo testo e il libro quarto del Filocolo viene recepito anche in Italia, come dimostra il fatto che Giolito nel 1553-1554 pubblica la Cuestiòn de Amor insieme alla traduzione in castigliano delle Tredici questioni ${ }^{24}$.

Questo tipo di pubblicazione continuerà a esistere e proliferare, riflettendo e veicolando, con lo scorrere dei decenni, una diversa concezione dell'amore $^{25}$. Libretti come Les Esguillons d'Amour, divisez en six Discours ${ }^{26}$, les Paradoxes d'amour du sieur de la Valletrye ${ }^{27}$, les Extravagances d'Amour, del 1604 (Paris, M. Guillemot), possono essere considerati come il risultato dell'evoluzione della pratica delle "questioni » e testimoniano di un interesse costante per l'analisi psicologica legata alla casistica amorosa, che arriva in certi casi a una finezza notevole ${ }^{28}$. Accanto

21. «Et ores que ne puisse parvenir a la melliflue eloquence d'Ulixes, m'est apres toute aultre perte demeure la plume pour a jamais persecuter le nom de la devoratrice de tout bon cueur, et que ainsi que soubz l'emprunte de Ibis le delicieux Ovide execra son mortel ennemy, je puis degrader soubz le nom de Circes l'inique imitatrice et heritiere des vices d'icelle, combien que quant a present me suffira d'avoir remonstre quatre especes d'amour couvertement celees soubz l'ancienne poesie, esperant cy apres en faire ung plus ample volume».

22. Per un approccio al vasto tema della fortuna di Ovidio nel Rinascimento si veda Bodo GuthmÜLler, Mito, poesia, arte. Saggi sulla tradizione ovidiana nel Rinascimento, Roma, Bulzoni, 1997.

23. Le Debat des deux Gentilzhommes Espagnolz sur le faict d'amour, Paris, Jehan Longis, 1541. Sulla Cuestiòn de Amor si veda il recente volume: DIEGO DE GUMIEL, Cuestión de amor, Valence, 1513, introduction, édition et notes de Françoise Vigier, Paris, Presses de la Sorbonne nouvelle, 2006.

24. Salvatore BongI, Annali di Gabriel Giolito de'Ferrari, Roma, 1890, vol. I, pp. 408-410.

25. Si veda l'elenco di opere evocate da G. REYNIER, Le roman sentimental avant l'Astrée, cit.

26. Les Esguillons d'Amour, divisez en six Discours, par L.D.G. Sr de Grivesne, Paris, A. du Brueil, 1599.

27. Les Paradoxes d'amour par le sieur de La Valletrye [S. 1.] : [s. n.], [s. d.] (quest'opera costituisce la seconda parte delle Secrettes ruses d'amour, Paris, A. di Breuil, 1611).

28. Si veda, per esempio, il quinto capitolo de Les Esguillons d'Amour, cit., consacrato alla gelosia. 
a questo tipo di trattatistica, assistiamo nel corso del Cinquecento allo sviluppo della novella, genere che riceve un impulso decisivo grazie al contemporaneo diffondersi della nuova traduzione del Decamerone voluta da Margherita di Navarra ${ }^{29}$. Brantôme coglierà il legame tra il piacere di raccontare e quello di disquisire: nel Recueil des Dames il «Discours sur les femmes mariées, les vefves et les filles, à sçavoir desquelles les unes sont plus chaudes à l'amour que les autres" riprende la IX questione del Filocolo, da lui definito «livre tant excellent ${ }^{30}$ » e la risolve a suo modo, cioè con attitudine ironica e dissacrante ${ }^{31}$.

\section{La traduzione integrale del romanzo}

Negli stessi anni in cui in Francia si impongono le Questioni d'amore, in Italia viene esaltato piuttosto, non a caso, il legame del Filocolo con l'epica. L'amore avventuroso dei due protagonisti ispira a un giovanissimo Lodovico Dolce una riduzione in ottave del romanzo ${ }^{32}$.

Ed è proprio in rapporto con lo sviluppo del genere romanzesco che va letta anche l'operazione della traduzione integrale del Filocolo di Adrien Sévin, con cui inizia la seconda fase della sua fortuna in Francia. Le Philocope viene pubblicato a Parigi nel 1542, presso l'editore Denis Janot, lo stesso che aveva pubblicato la terza edizione delle Treize elegantes demandes d'amour che ho descritto sopra. Janot è anche, com'è noto, l'editore dei primi fortunati libri degli Amadigi, traduzione del romanzo spagnolo che costituisce un "caso" editoriale nella Francia del XVI secolo, per

29. Antoine Le Maçon, 1545 chez Estienne Roffet dit le Faulcheur.

30. Recueil des Dames I, vii : "Ceste Marie fust celle Dame qui fut femme de Robert d'Artois, et aymée de Bocasse, qui pour lors florissoit, pour laquelle il escrivit en sa langue vulgaire ces deux livres tant excellens: La Flammette, et Le Philocope».

31. Cfr. L. SozzI, "Boccaccio in Francia nel Cinquecento", cit., pp. 259-260.

32. Cfr. A. BacCHI Della LegA, Bibliografia boccaccesca, cit., p. 107: "Il Filocolo ridotto in ottava rima da Lodovico Dolce. Venezia, per Bernardino de’Vitali, 1532. Edis. in $4^{\circ}$. Il Dolce nella sua prima gioventù ridusse questo romanzo del Certaldese in ottava rima, ed avendolo poi rassettato in età matura, sebbene non finito, ne diede fuori per le stampe i soli primi nove Canti». Romei, nella voce del $D B I$ parla invece di un rifacimento in ottave del Dolce del 1537, dal titolo L'amore di Florio e Biancofiore. Quest'opera rara, che non sono riuscita a consultare nelle biblioteche parigine (i cataloghi informatici la segnalano solo alla Marciana, segnatura: 84.C.89.1), non era sfuggita all'erudito segretario del Marquis de Paulmy, estensore del foglio manoscritto che accompagna l'in-folio della Biblioteca dell'Arsenal di Parigi dal titolo Le Philocope de Messire Jehan Boccace Florentin, contenant l'histoire de Fleury et Blanchefleur, Paris, Denis Janot, 1542 [RES. Fol. BL. 950]. In aggiunta ad altre varie notizie sul Filocolo, infatti, si legge: «Il [Filocolo] a été traduit de Prose en vers italiens par Ludovico Dolce». 
il successo strepitoso ${ }^{33}$. Il primo libro degli Amadigi viene pubblicato nel 1540, la traduzione di Sévin del Filocolo esce due anni dopo, con lo stesso formato (un elegante in-folio), con le stesse illustrazioni che, come è stato dimostrato da Jean-Marc Chatelain in uno studio consacrato agli Amadigi 34 erano inizialmente state previste per il Filocolo. Non solo: les pièces liminaires del Philocope sono firmate da Nicolas Herberay des Essart, cioè dell'autore della traduzione dei primi libri degli Amadigi; la sua devise, "Acuerdo Olvido", ricorre in entrambi i volumi; Adrien Sévin, inoltre, era parente di Michel Sévin, uno dei successivi traduttori degli Amadigi e autore di una prefazione importante per la teoria del romanzo in Francia nel ' $500^{35}$. Siamo dunque di fronte alla stesso prodotto editoriale, preparato dalle stesse persone, destinato allo stesso pubblico, cioè la nobiltà di corte. E'un fenomeno interessante di assimilazione, paragonabile a quello studiato da Daniel Javich a proposito dell'Orlando Furioso presentato a Venezia da Giolito nella stessa veste editoriale delle traduzioni dei poemi classici (con lo stesso formato, gli stessi paratesti, le stesse illustrazioni, le allegorie, il commento, il glossario) ${ }^{36}$. Secondo lo studioso americano lo scopo di Giolito era conferire all'Orlando furioso la dignità dei classici, trasporre su Ariosto l'autorità dei grandi poemi antichi come le Metamorfosi, l'Eneide, l'Iliade, l'Odissea, l'Achilleide. Nel caso di Janot l'affiliazione agisce in entrambi i sensi: gli Amadigi si vendevano bene, quindi presentare il Filocolo come identico prodotto era sicuramente una garanzia di successo. Ma il discorso resta valido - ed è anzi per noi più interessante - anche in senso inverso: il Filocolo era percepito dal pubblico francese come un grande classico della tradizione italiana: associarlo nella ricezione del pubblico agli Amadigi serviva a nobilitare il romanzo spagnolo, a conferirgli quello smalto di dignità culturale così rassicurante per il lettore di ogni epoca. Questa analisi delle scelte editoriali di Janot è per noi importante perché ci permette di affermare che il Filocolo era considerato come un grande classico, in grado di irradiare la propria autorità ad altre opere e di legittimarle.

33. Michel Simonin, "La disgrâce d'Amadis", in IDEM, L'encre et la lumière, Genève, Droz, 2004, pp. 189-234.

34. Jean-Marc Chatelain, "L'illustration d'Amadis de Gaule dans les éditions françaises du XVIe siècle", in Les Amadis en France au XVIe siècle, Paris, Editions rue d'Ulm, 2000, pp. 41-52.

35. Sergio CAPPELlo, "Il Discours sur les livres d'Amadis di Michel Sevin (1548)», in Il romanzo nella Francia del Rinascimento: dall'eredità medievale all'Astrea. Atti del Convegno Internazionale Gargnano (7-9 ottobre 1993), Fasano, Schena, 1996, pp. 207-224.

36. Daniel Javich, Ariosto classico. La canonizzazione dell'Orlando furioso, Milano, Bruno Mondadori, 1999. 
L'edizione del 1542 interessa anche un altro aspetto della cultura francese di questo periodo: l'importanza centrale delle traduzioni, tema fondamentale della politica culturale di Francesco I, e del rapporto con l'Italia ${ }^{37}$. Nell'Epistre du Translateur il traduttore dichiara di voler "contenter et donner recreation à tous ceulx qui desirent sçavoir l'une et l'autre langue, Italienne et Francoyse, en laquelle je voy plusieurs se delecter affectueusement ${ }^{38}$ ». Attorno alla traduzione sembra esserci poi una forte attesa: «Ie me suis mis à translater en nostre dicte langue le Philocope de l'elegant Iehan Boccace, non moins Poete qu'Orateur par expres commandement de monseigneur de Gyé vostre frere, mon bien facteur, et aux prieres de plusieurs mes bons amys, qui avoient grand desir de le veoir ${ }^{39}$ ". Questa traduzione doveva essere considerata dall'esordiente Adrien Sévin come la tappa necessaria per mettersi in luce e far conoscere i propri meriti di narratore. Sceglie infatti la sede dell'Epistre du translateur per inserire in modo un po' inatteso - una novella scritta di suo pugno, che poco ha a che fare con il Filocolo, se non per il fatto, in realtà più che ovvio, che racconta una storia d'amore contrastato. Il dato invece molto interessante è che l'intrigo della novella ricorda sorprendentemente quello di Romeo e Giulietta. Questo aspetto della prefazione di Sévin, sul quale si è già espresso Hauvette fin dal 1921 - e che non ignoravano gli studiosi di Shakespeare, come Hauvette stesso ricorda ${ }^{40}$, costituisce una prova ulteriore delle aspettative del traduttore e dell'editore che, evidentemente, avevano investito molto su questo volume.

Le scelte di Janot (formato, illustrazioni) dimostrano anche che la traduzione del Filocolo era stata concepita con un'attenzione particolare al pubblico femminile che, si sa, in questi anni diventa una delle mire su cui più si concentrano gli sforzi degli editori. In effetti il romanzo di Boccaccio aveva fin nelle sue intime fibre tutti gli elementi necessari per attirare le lettrici: scritto per richiesta della donna amata, era dedicato dal

37. Del fatto che le opere del Boccaccio venivano usate nel corso del Cinquecento per l'apprendimento dell'italiano offre testimonianza, per esempio, l'edizione bilingue della Fiammetta di L'Angelier del 1585 (cfr. Jean BALSAMO \& Michel SimOnin, Abel L'Angelier et Françoise de Louvain, Genève, Droz, 2002, p. 203).

38. Le Philocope de Messire Jehan Boccace Florentin, cit., feuillet III.

39. Ibidem.

40. Henri Hauvette, Une variante française de la légende de Roméo et Juliette, in "Revue de Littérature Comparée», I, 1921, pp. 329-337. Hauvette segnala l'ambientazione orientale, "esotica" della novella e la scelta bizzarra dei nomi, quasi illeggibili, dei protagonisti: Halquadrich e Burglipha. Lo studioso analizza anche l'influenza della novella di Sévin sul Roméo et Juliette di Ducis, partendo da precise circostanze testuali : uno studio che apre la riflessione su inediti percorsi di influenze che possono portare molto lontano. 
narratore alle donne, per il loro divertimento e anche per la loro educazione alla fedeltà in amore ${ }^{41}$.

L'ultimo aspetto su cui vorrei soffermarmi riguarda la presenza dei titoli in margine, che invece sono assenti nelle edizioni del 1540 e del $1542 \mathrm{degli}$ Amadigi. Oltre a segnalare i vari episodi del romanzo, le «manchettes», sottolineano tutti i riferimenti mitici, le «belles sentences» e le «belles similitudes».

Questa scelta dell'editore suggerisce che il libro fosse destinato a essere usato anche come repertorio mitologico, retorico e stilistico. La fama di erudito che accompagnava Boccaccio e la vicinanza contenutistica ad Ovidio (senz'altro la fonte più sfruttata ${ }^{42}$, oltre ad essere l'autore del libro

41. Giovanni Boccaccio, Filocolo, ed. A.E. Quaglio, in Tutte le opere di Giovanni Boccaccio, a cura di V. Branca, vol. I, Milano, Mondadori, 1967, p. 67 (I, ii, 3-6) : «E voi, giovinette amorose, le quali ne' vostri dilicati petti portate l'ardenti fiamme d'amore più occulte, porgete le vostre orecchi con non mutabile intendimento a'nuovi versi: li quali non vi porgeranno i crudeli incendimenti dell'antica Troia, né le sanguinose battaglie di Farsaglia, le quali nell'animo alcuna durezza vi rechino; ma udirete i pietosi avvenimenti dello innamorato Florio e della sua Biancifiore, li quali vi fieno graziosi molto. E, udendoli, potrete sapere quanto ad Amore sia in piacere il fare un giovane solo signore della sua mente, sanza porgere a molti vano intendimento, però che molte volte si perde l'un per l'altro, e suolsi dire che chi due lepri caccia, talvolta piglia l'una e spesso non niuna. Dunque apprendete d'amare uno solo, il quale ami voi perfettamente, sì come fece la savia giovane la quale per lunga sofferenza Amore recò al disiato fine. E se le presenti cose, o voi, giovani e donzelle, generano ne' vostri animi alcun frutto e diletto, non siate ingrati di porgere divote laudi a Giove e al nuovo autore». Nell'ultima pagina della traduzione del 1542, Adrien Sévin riprende a sua volta questi temi. Cfr. Le Philocope de Messire Jehan Boccace Florentin, cit., feuillet CLXXIIII $v^{\circ}$ : «Le translateur pour conclusion. Mon petit œuvre et gratieux labeur, le vent paisible soufle desormais ton vaisseau es seurs rivages: car les autres ventz nuisibles n'ont puissance contre tes voiles. Prens doncques port et te rends diligemment es delicates mains de la tres vertueuse et seul Phenix, des dames, qui par aventure te recevra, et employera ses beaulx yeulx à te veoir, de sorte que tu te pourras dire eternellement glorieux. Et si ainsi est, n'ayes soing d'estre ailleurs, ains revele moy ton immerité recueil, à ce que cy apres ie me hazarde de t'acompaigner d'autre livre, non pareil aux beaulx vers de Virgile et de Lucan, qui traictent des fieres armes de Mars, mais en suyvant les amours d'Ovide que ie doibz honorer reveremment, et n'entreprendre trop haulte matiere à ma honte. Car la Cigoigne qui demeure aux grans palais et haultes tours descend bien pour boire au petit fleuve. Pareillement Alcion en volant bat ses aesles sur les undes salées. Aussi ce m'est assez de povoir plaire avec petis moyens et continuelz services à vous ma tres redoubtée dame, et que mon debile et rural esperit vous puisse quelque foiz contenter par son labeur, excusant les faultes, sans donner occasion à plusieurs de me reprendre et estre mocqué iustement d'eulx, lesquelz ie prie avoir esgard à mon intention: supplyant le seigneur dieu conduire icelle à sa totalle louange et a vostre passetemps honeste». Sul topos della scrittura richiesta da una donna, in un ambiente nutrito delle stesse letture si veda Marie-Françoise PIÉJUS, «Une traduction française de la Historia de duobus amantibus d'Eneas Silvius Piccolomini", in La circulation des hommes et des oeuvres entre la France et l'Italie à l'époque de la Renaissance, Actes du Colloque International, Paris, 22-24 novembre 1990, Paris, Université de la Sorbonne Nouvelle, 1992, p. 108.

42. Antonio Enzo Quaglio, Tra fonti e testi del "Filocolo", in "Giornale storico della letteratura italiana», 139 (1962), pp. 321-369, 513-540. 
"galeotto" - l'Ars Amatoria - che ispira l'amore tra i protagonisti ${ }^{43}$ ) motivano sufficientemente la destinazione di repertorio del Filocolo nella Francia degli anni' 40.

Modello di narrazione (cornice, ecc.), modello linguistico-retorico, serbatoio di riflessioni amorose, il Filocolo rimane un testo di riferimento in Francia per tutto il XVI secolo. Sicuramente era conosciuto: possiamo trovare una prova della sua notorietà, per esempio, in una traduzione del Tasso dell'inizio del '600. Jean Baudoin, il traduttore dei Discorsi del Tasso $^{44}$, nella prefazione alla traduzione del Discorso della Gelosia, asserisce di voler tradurre in francese unicamente quello che il suo pubblico può riconoscere e apprezzare ${ }^{45}$. Tralascia quindi nella sua versione i nomi e in alcuni casi le citazioni di poeti come Dante e Della Casa, cita invece sempre Petrarca e cita dunque interamente il passo del Filocolo sulla dimora della gelosia utilizzato dal Tasso ${ }^{46}$ : questa scelta prova che il romanzo di Boccaccio era considerato come un'opera immediatamente identificabile per il pubblico e anche che era usato correntemente come repertorio di immagini mitiche.

La reputazione negativa di romanzo noioso, scritto giovanile, troppo lungo, con troppe digressioni che, di fatto, accompagna per secoli il Filocolo, finirà tuttavia per imporsi anche in Francia. L'estensore dell'edizione settecentesca del La Croix du Maine, infatti, afferma:

Quelques-uns, si l'on en croit le Muzio au commencement de ses Battaglie, ont dit que Bocace préféroit son Filocope à tous ses autres Ouvrages, même au Décaméron, en quoi il auroit marqué bien peu de goût. C'est en effet un Roman très ennuyeux, soit par les choses imaginées, soit par la manière de les raconter. On y voit un mélange extravagant, du Paganisme avec le Christianisme. Junon, tout à l'entrée, y descend du Ciel, pour aller trouver le Pape, son Vicaire Général, à qui, auparavant elle envoie sa messagere Iris, la figlia di Taumante, l'avertir de sa venue. Le style y est empoulé par

43. Filocolo, ed. cit., p. 123 (I, 45, 6) : «E loro in brieve termine insegnate conoscer le lettere, fece loro leggere il santo libro d'Ovidio, nel quale il sommo poeta mostra come i santi fuochi di Venere si deano ne' freddi cuori con sollecitudine accendere».

44. Les Morales de Torquato Tasso, où il est traitté de la Court, de l'Oisiveté, de la Vertu des dames illustres, de la Vertu heroyque, du Mariage, de la Jalousie, de l'Amour, de l'Amitié, de la Compassion, et de la Paix; traduictes par J. Baudoin, Paris, Augustin Courbé, 1632.

45. Les Morales..., cit., Aux bons esprits (pagine non numerate): "J'ay donc mis la main à l'œuvre, avecque dessein de m'exprimer nettement, d'ajuster sa façon d'escrire à la nostre, et d'en choisir les plus beaux endroits, sans m'attacher ny aux noms propres Italiens, ny aux particularitez estrangeres, ny aux choses que j'ay jugé ne pouvoir estre dittes agreablement, qu'en la propre langue de leur Autheur".

46. La fonte del passo del Filocolo (III, 24) è Ovidio, Met. II, 752-832. 
tout et affecté jusqu'au rudicule, comme, lorsque parlant de l'Église appelée à Naples La Chiesa dello Spirito Santo, au lieu de dire simplement l'Église du S. Esprit, il a recours à la paraphrase, et dit, le Temple du Prince de tous les Saints Oiseaux, il Tempio del Principe et di tutti i Sancti Uccelli. Le Muzio a donc raison de croire que si Bocace, dans sa première jeunesse, a eu quelque complaisance pour son Filocope, il changea de sentiment, lorsqu'un âge plus mûr lui eût raffiné le goût, témoin les deux nouvelles si traînantes dans le Filocope, retouchées depuis avec tant de précision et de justesse dans le Decameron ${ }^{47}$.

Il segretario del Marchese di Paulmy che redige la nota manoscritta che accompagna l'esemplare del Philocope del 1542 della Biblioteca dell'Arsenal ${ }^{48}$, a sua volta riporta quella che nella seconda metà del Settecento era ormai communis opinio: "On accuse cette histoire d'être trop prolixe et ce n'est pas l'ouvrage de Boccace qui se fait lire avec le plus de plaisir». Del resto la degradazione della reputazione del Filocolo è ravvisabile già nel corso del Cinquecento, in modo fortemente simbolico, nel contesto italiano. Se all'inizio del secolo infatti «le questioni» letterariamente consacrate da Boccaccio vengono evocate da Castiglione come prassi cortese quotidiana, alla fine del secolo, l'altro trattato di comportamento destinato a dettare legge nell'aristocratico mondo delle corti europee, Il Galateo, suggerisce al proprio lettore di evitare «il favellar pomposo » del Filocolo, "spiacevole e tedioso ad udire» (cap. XXIII). Il giudizio paradossale di Ortensio Lando citato in apertura finirà per imporsi nell'immaginario: tramontata l'epoca delle «belle quistioni», stemperato il successo degli Amadigi, del romanzo di Florio e Biancofiore verranno ricordati soprattutto i difetti e nessun editore francese avrà, dopo il Cinquecento, l'audace idea di ripubblicarlo.

47. Girolamo MuZIO, Battaglie per difesa dell'italica lingua, a cura di R. Sodano, Torino RES, 1994, p. 5-6.

48. Le Philocope de Messire Jehan Boccace Florentin, cit. 